\title{
Multi-Disciplinary Interventions for Children and Adolescents with Obesity: the Whānau Pakari Experience ${ }^{\dagger}$
}

\author{
Lisa E. Wynter * and Niamh A. McCormack \\ Department of Paediatrics, Taranaki District Health Board, New Plymouth 4310, New Zealand; \\ Niamh.McCormack@tdhb.org.nz \\ * Correspondence: lisa.wynter@tdhb.org.nz \\ + Presented at the 2019 Annual Meeting of the Nutrition Society of New Zealand, Napier, New Zealand, \\ 28-29 November 2019.
}

Published: 18 March 2020

Background/Objectives: Multi-disciplinary interventions remain recommended best practice for management of childhood obesity. The purpose of Whānau Pakari (WP) was to create a multi-disciplinary assessment and intervention programme for children/adolescents with obesity in Taranaki, incorporating a randomised clinical trial (RCT).

Methods: The programme was a collaboration between Taranaki DHB and Sport Taranaki. It specifically focusses on those over-represented in obesity statistics, namely Māori and those from most deprived households, thereby addressing health inequities. An RCT ( $\mathrm{n}=102$ in intervention group, $\mathrm{n}=101$ in control group) was embedded within the service, offering holistic, 6-monthly, home-based assessments and advice (control), or assessments and weekly group sessions for 12 months. The primary outcome was the change in BMI standard deviation score (SDS) at 12 months. Secondary outcomes included changes in health-related quality of life (HRQOL), and physical activity (550-m walk/run time).

Results: Enhanced levels of engagement with at-risk groups were achieved. Engagement with WP was associated with a significant decrease in BMI SDS in the low-intensity control $(-0.12,95 \% \mathrm{CI}$ -0.2 to -0.03$)$ and high-intensity intervention $(-0.10,95 \% \mathrm{CI}-0.19$ to -0.02$)$ at 12 months. Attendance $\geq 70 \%$ in the high-intensity intervention doubled the effect $(-0.22,95 \%$ CI -0.36 to $-0.09, p=0.04)$. Improvements occurred in HRQOL and cardiovascular fitness in both groups. Multi-source programme evaluation found high stakeholder, referrer and participant satisfaction. Economic evaluation demonstrated Whānau Pakari was cost-effective compared with the previous model.

Key take-home messages/Discussion: WP is an acceptable, appropriate intervention for children/youth with obesity that has improved health inequity in service delivery, and weight status at 12 months. It is a unique one-team model of care providing continuity, with a comprehensive assessment that identifies and manages weight-related comorbidities, and assists in planning individualised interventions.

(C) 2020 by the authors. Licensee MDPI, Basel, Switzerland. This article is an open access article distributed under the terms and conditions of the Creative Commons Attribution (CC BY) license (http://creativecommons.org/licenses/by/4.0/). 